\title{
DOSIS DE GONADOTROPINAS (eCG Y hCG), SUPEROVULACIÓN Y OBTENCIÓN DE EMBRIONES EN ALPACAS
}

\author{
César Novoa $^{1}$, Enrique Franco ${ }^{2}$, Wilber García ${ }^{2}$ y Danilo Pezo ${ }^{2}$
}

\section{Abstract}

Research on the effect of different doses of gonadotropins on superovulation in Huacaya alpacas is reported. Adult estral females (3-6 years old) with ovarian folicles less than $7 \mathrm{~mm}$ in diameter, as ascertained by ultrasonography, were utilized. The treatments were: T1) $\mathrm{n}=6$; day $0: \mathrm{eCG}, 750 \mathrm{iu}$, im; day $5: \mathrm{hCG}, 750 \mathrm{iu}, \mathrm{mi}, \mathrm{T} 2) \mathrm{n}=6$; day 0: e CG, $500 \mathrm{iu}$, im; day 5: hCG, $750 \mathrm{iu}$, im and T3) $\mathrm{n}=6$; day 0: eCG, $750 \mathrm{iu}, \mathrm{mi}$; day 5: hCG, $1000 \mathrm{iu}, \mathrm{im}$. In each of these treatments, the hormones were injected at 07:00 hours and on day 5, the females were mated twice by intact males, at 07:00 and 17:00. Confirmation of ovulation and embryo collection was done by mid-ventral laparotomy, carried out as follows: $\mathrm{A}$ ) in $\mathrm{T} 1$ and $\mathrm{T} 2,50 \%$ of the females were done on day 7 and the remainder on day 8 post-injection of hCG and B) in T3, all females were done on day 3 , after hCG injection. The number of ovulations in T3 $(12.6 \pm 1.8)$ was significantly higher $(\mathrm{p}<.10)$ than in $\mathrm{T} 2(6.0 \pm 2.8)$ but it did not differ from results of $\mathrm{T} 1$ $(6.8 \pm 2.6)$ (p>.10). Embryos were collected only from females in T3 (31/126). Doses of 500 and $750 \mathrm{eCG}$ iu and $750 \mathrm{hCG}$ iu effectively induced superovulation in alpacas, but the embryo collection method must be imporved in order to increase the recovery rate. Key Words: superovulation, alpaca.

\section{Resumen}

Se estudió el efecto de dosis de gonadotropinas sobre la superovulación en alpacas, también la colección de embriones a diferentes periodos post monta natural. El estudio se realizó en la estación de investigaciones de Maranganí-La Raya, del IVITA (Instituto Veterinario de Investigaciones Tropicales y de Altura), ubicado en Cuzco, a $4200 \mathrm{msnm}, 71^{\circ}$ latitud sur y $71^{\circ}$ longitud oeste; se usaron hembras adultas ( 3 a 6 años de edad) de la raza Huacaya, que estaban en celo y que al examen ovárico por ultrasonografía, tenían folículos menores de $7 \mathrm{~mm}$ de diámetro. Los tratamientos fueron: T1) n =6; día 0 : 750 ui, im de eCG; día 5: 750 ui, im de hCG, T2) n = 6; día 0: 500 ui, im de eCG; día 5: 750 ui, im de hCG y T3) n = 6; día 0: 750 ui, im de eCG; día 5: 1000 ui, im de hCG. En los tres casos las hormonas fueron inyectadas a las 7:00 horas y las hembras fueron servidas con macho entero, el día 5, dos veces, a las 7:00 y 17:00 horas. El registro de ovulaciones y la colección de embriones se hicieron mediante laparotomía ventro medial, como sigue: a) en $\mathrm{T} 1$ y T2, el $50 \%$ de animales fue intervenido a los $7 \mathrm{~d}$ y el resto a los $8 \mathrm{~d}$ post inyección de hCG y b) en T3, todos los animales fueron intervenidos a los $3 \mathrm{~d}$ de dicha inyección. El número de ovulaciones en T3 (12.6 
$\pm 1.8)$ fue significativamente mayor $(\mathrm{P}<.10)$ comparado con $\mathrm{T} 2(6.0 \pm 2.8)$ pero no con $\mathrm{T} 1$ (6.8 \pm 2.6$)$. Sólo en T3 se recuperaron embriones (31/126), esto es, cuando la colección se hizo 3 días después de la inyección de hCG. Se concluye que, la dosis de eCG entre 500 y 750 ui y de hCG de 750 ui, son apropiadas para inducir ovulación múltiple en alpacas. El método de colección de embriones empleado, requiere de algunos ajustes para mejorar su eficiencia.

Palabras clave: Superovulación, alpaca.

\section{Tniroduccion}

La mayoría de la población de alpacas y llamas en el país se cría en rebaños pequeños, de composición heterogénea, que incluyen animales de diferentes colores y de razas (alpacas Huacaya y Suri y llamas Chaku y Ccara) y sus cruces. En estas condiciones los diferentes recursos genéticos se encuentran sub utilizados, su potencial productivo que podría ser mejorado mediante selección se ve muy limitado. Para superar estas limitaciones varias instituciones están trabajando en la formación de núcleos genéticos de alpacas y llamas "puras" $\mathrm{y}$, con base en estos animales preseleccionados, avanzar en el mejoramiento genético mediante selección de los animales que reúnen características deseables desde el punto de vista productivo. El objetivo es lograr que la ganancia genética acumulada en dichos núcleos, sea transmitida a los rebaños de los productores en forma de vientres y reproductores mejorados.

Mientras dichos esfuerzos se van materializando y ampliando, se necesita desarrollar técnicas reproductivas que faciliten la difusión masiva de dichos animales mejorados. En la actualidad, el número promedio de crías $(n=6)$ que una hembra alpaca o llama puede producir durante toda su vida reproductiva es muy limitado para difundir material genético deseado. Las técnicas de la ovulación múltiple (OM) y transferencia de embriones (TE) son una posibilidad para superar esta limitación; permitirían, por otro lado, reducir el intervalo generacional mediante el uso de animales jóvenes.

Los estudios sobre OM y TE en alpacas y llamas son escasos. Para estimular el crecimiento folicular se ha utilizado la gonadotropina coriónica equina (eCG) o la hormona estimulante del crecimiento folicular (FSH) y luego para inducir la ovulación múltiple se ha empleado ya sea la gonadotropina coriónica humana (hCG), la hormona liberadora de gonadotropina (GnRH) o la monta natural. Estos tratamientos han sido aplicados ya sea en fase folicular (Novoa y Sumar 1968; Palomino et al.,1987; Bravo et al.,1995) o en fase Progestacional inducida (Bourke $e t$ al., 1992, 1994; Correa et al., 1994). En general los tratamientos indicados, tanto en fase folicular como progestacional, resultaron en una respuesta ovulatoria variable, el número de ovulaciones varió entre 2 a 11. En aquellos ensayos en que se usó monta natural para inducir la superovulación, la respuesta fue nula o baja, comparada con aquella en que se usó hCG o GnRH. Recientemente, Velásquez y Novoa (1999) probaron el uso de 1000 ui de eCG seguida de 1000 ui de hCG, tanto en fase folicular como Progestacional inducida, obteniendo $8.2 \pm 2.6$ y $17.8 \pm 8.3$ ovulaciones, respectivamente. Esta diferencia fue significativa $(\mathrm{P}<, 05)$; sin embargo la respuesta ovárica fue excesiva, particularmente, en el tratamiento en fase progestacional, lo que determinó que el ovario quede atrapado en la bolsa ovárica. Con tales antecedentes este estudio se 
planteó los siguientes objetivos:1)probar dos dosis de eCG (500 y 750 ui) y dos dosis de hCG (750 y 1000 ui) y su efecto sobre la superovulación en alpacas y 2) Colectar embriones por medios quirúrgicos a los 3,7 y 8 d. pos inyección de hCG

\section{Material y Métodos}

El estudio se realizó entre abril y mayo de 1997, en la Estación Experimental de Maranganí - La Raya del Instituto Veterinario de Investigaciones Tropicales y de Altura (IVITA) de la Universidad Nacional Mayor de San Marcos. La Estación se encuentra a $4,200 \mathrm{msnm}, 71^{\circ}$ latitud sur y $71^{\circ}$ longitud oeste en el Departamento del Cusco.

\section{Animales y Manejo}

Se utilizaron 22 alpacas de la raza Huacaya (3 a 6 años de edad) de 55 a $60 \mathrm{Kg}$. de peso vivo, que se encontraban en celo y que al examen ovárico mediante ultrasonografía mostraron un desarrollo folicular menor de $7 \mathrm{~mm}$ de diámetro. El examen de celo se hizo por observación de la conducta en presencia de macho y la inspección ovárica mediante un ecógrafo Bmode, Aloka, SSD-210 Dx, con dispositivo de aplicación rectal (I shape, $5 \mathrm{mhz}$ de frecuencia). Durante el estudio las hembras fueron alimentadas en pasturas cultivadas, una mezcla de Tripolium repens y Lolium perenne y estuvieron separadas de machos enteros con la excepción del día en que recibieron servicios controlados, de acuerdo al plan de tratamiento que se describe a continuación.

\section{Tratamientos}

Los tratamientos hormonales fueron los siguientes:

T1) $n=6$; día 0: 750 ui, im de eCG (Folligon, intervet. int. Holanda); día 5: 750 ui, im de hCG (Pregnyl, Química Suiza)

T2) n= 6; día 0: 500 ui, im de eCG; día 5: 750 ui, im de hCG

T3) n= 6; día 0: 750 ui, im de eCG; día 5: 1000 ui, im de hCG

En los tres casos la inyección hormonal se aplicó en una sola dosis a las 7.00 horas y las hembras fueron servidas con macho entero el día 5, dos veces, a las 7.00 y 17.00 horas.

\section{Registro de Ovulaciones y Colección de Embriones}

Se realizó una laparotomía a través de la línea media ventral, de acuerdo al procedimiento descrito por Dietz et al. (1975), luego los ovarios fueron expuestos procediéndose a contar el número de cuerpos luteos y a continuación se realizó la colección de embriones.

Con el objeto de obtener información colateral sobre el estadio de desarrollo de los embriones, la intervención quirúrgica y lavado del tracto reproductivo para recuperar embriones se ejecutó como sigue: en T1 y $\mathrm{T} 2,50 \%$ de animales a $\operatorname{los} 7 \mathrm{~d}$. y el otro 50 $\%$ a los 8 d post inyección de hCG y en el T3 a los 3 días después de dicha inyección.

El lavado del tracto reproductivo se realizó con solución salina fisiológica (60ml.) ingresando por el infundíbulo y colectando el líquido del lavado mediante una pipeta pasteur, cuyo extremo más angosto, había sido insertado en el cuerno uterino. Para asegurar que el líquido pase a través de la pipeta fue necesario obliterar el cuerno uterino con una pinza, aplicada en la parte media del cuerno. Los embriones fueron localizados mediante un microscopio usando 30 de aumento, se anotó el número recuperado y su estadío de desarrollo 


\section{Análisis de Resultados}

El número de cuerpos lúteos, usado como parámetro para medir la respuesta ovulatoria, fue sometido a análisis de variancia y la comparación entre promedios se realizó mediante la prueba de Duncan (Steel and Torrie, 1990). El modelo lineal empleado fue el siguiente: $y i j=u+T i+E i j ;$ donde $y i j=o b s e r v a c i o ́ n$ individual, $\mathrm{u}=$ media poblacional, $\mathrm{i}=$ tratamientos $1,2,3$, y Eij= error experimental.

En el caso de los embriones se describe el número recuperado del oviducto izquierdo y derecho y su estadío de desarrollo y el porcentaje total de recuperación.

\section{Resultados y Discusión}

Los resultados sobre la respuesta ovulatoria se muestran en el Cuadro 1 y los relacionados con la colección de embriones en el Cuadro 2. Respecto al número de ovulaciones, las diferencias observadas entre tratamientos no fueron significativas con la excepción de T2 $(6.0 \pm 2.8)$ que fue significativamente menor $(\mathrm{P}<.10)$ que $\mathrm{T} 3$ (12.6 \pm 1.8$)$. Esta diferencia probablemente se deba a la dosis de eCG y no de hCG empleadas ya que los resultados de T3 vs T1 no fueron diferentes; en general estos resultados son consistentes con los obtenidos previamente (Velásquez y Novoa 1999), con la diferencia que en este caso la respuesta ovulatoria fue más moderada en número de ovulaciones así como en el número de folículos quísticos (>de $13 \mathrm{~mm}$ de diámetro); sólo un animal en T1 mostró 3 folículos quísticos. Aunque los animales tratados tuvieron folículos menores de $7 \mathrm{~mm}$ de diámetro la respuesta ovulatoria individual fue variable y similar a la registrada en otros estudios (Velásquez y Novoa, 1999; Bravo et al., 1995). Esto probablemente se deba a la variación en desarrollo folicular, presente en los animales al momento que recibieron la inyección gonadotrópica. En vacas, Monniaux et al. (1983) demostraron que la mejor respuesta ovulatoria se obtiene cuando en el ovario se encuentra una población suficiente de folículos pequeños ( $=2 \mathrm{~mm}$ de diámetro). En vacas la inyección de eCG en la mitad de la fase luteal ( 8 a 12 d) del ciclo, provoca la mayor respuesta ovárica debido a que en ese momento contiene el mayor número de folículos antrales de pequeño y mediano tamaño. Con respecto a la colección de embriones, sólo en T3 se recuperaron 31 de un total de 126 embriones potenciales (es decir 126 ovulaciones) y se encontraban en el estadío de 2 a 8 bastómeros. En éste caso la colección se realizó 3 d después de la inyección de hCG; en cambio en T1 y T2 la colección se efectuó los a 7 y 8 d después de dicha inyección y la recuperación fue cero embriones. A pesar que la técnica de colección fue la misma en los tres casos, no se tiene una explicación clara para el fracaso en la recuperación de embriones en T1 y T2. Observaciones previas, que requieren verificación, indicarían que el desarrollo embrionario en alpacas es más rápido que en otros rumiantes, de tal suerte que a los 7-8 d post inseminación (i.e post inyección de hCG) los embriones se encontrarían en el estadio de blastocito expandido, es decir libre de la zona pelúcida. En esta condición los embriones pueden ser fácilmente dañados por la manipulación del lavado $\mathrm{y}$, por lo tanto, imposibilitar su recuperación.

El hecho de no haber recuperado óvulos sin fertilizar indicaría que el régimen de monta utilizado es el apropiado para lograr la fertilización masiva de óvulos. Esto sin embargo requerirá confirmación en la medida que se mejore la eficiencia en la colección de embriones. 
Cuadro 1. Respuesta ovulatoria (cuerpos luteos) en alpacas tratadas con diferentes dosis de ecg y hcg.

\begin{tabular}{|c|c|c|c|c|c|c|c|}
\hline & \multirow[t]{2}{*}{ Tratamientos } & \multirow{2}{*}{$\begin{array}{l}\text { Hembras } \\
(\mathrm{n})\end{array}$} & \multicolumn{5}{|c|}{ Cuerpos Luteos (n) } \\
\hline & & & $\begin{array}{l}\text { Ovario } \\
\text { Izquierdo }\end{array}$ & $\begin{array}{l}\text { Ovario } \\
\text { Derecho }\end{array}$ & Total & $\bar{x} \pm 5$ & Rango \\
\hline T1: & $\begin{array}{l}\text { Dia o; eCG, } 750 \text { ui, im } \\
\text { Dia 5; HcG, } 750 \text { ui, im }\end{array}$ & 6 & 20 & 21 & 41 & $6.8 \pm 2.6$ & $1-18$ \\
\hline $\mathrm{T} 2:$ & $\begin{array}{l}\text { Dia 0; eCG, } 500 \text { ui, im } \\
\text { Dia 5; hCG, } 750 \text { ui, im }\end{array}$ & 6 & 14 & 22 & 36 & $6.0 \pm 2.8$ & $1-18$ \\
\hline T3: & $\begin{array}{l}\text { Dia 0; eCG, } 750 \text { ui, im } \\
\text { Dia 5; hCG, } 1000 \text { ui,im }\end{array}$ & 6 & 62 & 64 & 126 & $12.6 \pm 1.8$ & $5-21$ \\
\hline
\end{tabular}

T2 Vs T3 $(\mathrm{P}<.10)$

Cuadro 2. Ovulaciones (cuerpos lúteos) y embriones colectados en alpacas tratadas con 750 ui, eCG (dia 0) y 1000 ui, hCG (dia 5).

\begin{tabular}{|c|c|c|c|c|c|c|c|}
\hline \multirow{3}{*}{ Hembra } & \multicolumn{3}{|c|}{ Cuerpos Lúteos (n) } & \multicolumn{4}{|c|}{ Embriones Colectados (n) } \\
\hline & \multicolumn{3}{|c|}{ Ovario } & \multicolumn{3}{|c|}{ Oviducto } & \multirow{2}{*}{$\%^{2}$} \\
\hline & Izq. & Der. & Total & Izq. & Der. & Total & \\
\hline 1 & 6 & 2 & 8 & $2(4 \mathrm{cel})^{3}$ & $2(8 \mathrm{cel})$ & 4 & 50 \\
\hline 2 & 9 & 10 & 19 & 0 & 0 & 0 & 0 \\
\hline 3 & 9 & 6 & 15 & $5(8 \mathrm{cel})$ & $4(8 \mathrm{cel})$ & 9 & 60 \\
\hline \multirow[t]{3}{*}{4} & 12 & 9 & 21 & $2(2 \mathrm{cel})$ & $2(4 \mathrm{cel})$ & & \\
\hline & & & & $1(4 \mathrm{cel})$ & & & \\
\hline & & & & $3(4 \mathrm{cel})$ & & 8 & 47 \\
\hline 5 & 5 & 5 & 10 & $1(8 \mathrm{cel})$ & 0 & 1 & 10 \\
\hline 6 & 9 & 11 & 20 & $6(4 \mathrm{cel})$ & 0 & 6 & 30 \\
\hline 7 & 1 & 4 & 5 & 0 & $2(8 \mathrm{cel})$ & 2 & 40 \\
\hline 8 & 3 & 8 & 11 & 0 & 0 & 0 & 0 \\
\hline 9 & 2 & 3 & 5 & 0 & 1 (8cel) & 1 & 20 \\
\hline 10 & 6 & 6 & 12 & 0 & 0 & 0 & 0 \\
\hline TOTAL & 62 & 64 & 126 & 20 & 11 & 31 & 25 \\
\hline $\begin{array}{ll}1 & \text { Colecció } \\
2 & \text { Total de }\end{array}$ & $=$ & 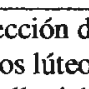 & & & & & \\
\hline
\end{tabular}




\section{Conclusiones}

Se concluye que las dosis de eCG entre 500 y 750 ui y de hCG de 750 ui, son las apropiadas para inducir la ovulación múltiple en alpacas, tanto en cantidad como en consistencia suficientes para cosechar y difundir material genético deseado. El procedimiento quirúrgico empleado para la colección de embriones, con algunos ajustes para mejorar su eficiencia, abre el camino para avanzar en la transferencia de embriones:

\section{Literatura Citada}

1. Bravo, W., T. Tsutsui y L. Lasley. 1995. Dose response to equine chorionic gonadotropin and subsequent ovulation in llamas. Small Ruminant Research 18: 157- 163

2. Bourke, D.A, C.L. Adam, C.E.Kile, P. Young y T.G. McEVoy. 1992. Superovulation and embryo Transfer in the llama. Proc. Ist. Int. Camel Conf.183185

3. Bourke, D.A., C.L. Adam, C.E. Kile, P. Young y T.G. Mc Evoy. 1994. Ovarian Responses To Pmsg and FSH in Llama. European Symp on South American Camelids, 75-81

4. Correa J.E., M. Ratto y R. Gatica. 1994. Actividad estral y Respuesta ovárica en alpacas y llamas tratadas con Progesterona y Gondotropina Arch. Med. Vet. 26:59-64

5. Dietz, O., F. Sanchez, H. Scheleiter y R. Teuscher. 1975. Operaciones y Anestesia de los animales pequeños y grandes Ed. Acribia Zaragoza España $556 \mathrm{p}$.

6. Novoa, C. y J. Sumar. 1968. Colección de huevos in vivo y ensayos de transferencia en alpacas. III Boletín Extraordinario, IVITA, UNMSM pp 3134

7. Monniaux, D., D. Chupin y J. Saumande. 1983. Superovulatory responses of cattle. Theriogenelogy 19: 55-81.

8. Palomino H., L. Tabacchi, E. Avila y O. Li. 1987. ensayo preliminar de transferencia de embriones en Camélidos Sudamericanos. Rev. Cam. Sudam, IVITA (Perú) 5:10-17.

9. Steel R.G.D. y J.H. Torrie. 1980. Principles and procedures of statistics, A. biometrical Approach. Mc Graw-Hill Book Company 2 ed., New York 633p.

10. Velásquez, C. y C. Novoa. 1999. Superovulación con PMSG aplicada en fase folicular y fase luteal en Alpacas. Rev. Inv. Vet. del Perú 10 (1): 39-47 